\title{
Almost Everywhere Convergence of Riesz Means Related to Schrödinger Operator with Constant Magnetic Fields
}

\author{
Liurui Deng ${ }^{1}$ and Bolin $\mathrm{Ma}^{2}$ \\ ${ }^{1}$ College of Finance and Statistics, Hunan University, Changsha 410082, China \\ ${ }^{2}$ College of Science and Information Engineering, Jiaxing University, Jiaxing, \\ Zhejiang 314001, China
}

Correspondence should be addressed to Bolin Ma; blma@mail.zjxu.edu.cn

Received 25 September 2012; Accepted 28 January 2013

Academic Editor: Chuanzhi Bai

Copyright (C) 2013 L. Deng and B. Ma. This is an open access article distributed under the Creative Commons Attribution License, which permits unrestricted use, distribution, and reproduction in any medium, provided the original work is properly cited.

We study almost everywhere convergence for Riesz means related to Schrödinger operator with constant magnetic fields. Through researching the weighted norm estimates for the maximal operator with power-weight functions, we obtain the desired result, which is similar to the work given by Anthony Carbery, Jose L. Rubio de Francia, and Luis Vega.

\section{Introduction}

The magnetic Schrödinger operator (MSO) with constant magnetic fields $H_{b}$ in $\mathbb{R}^{n}$ is of the form

$$
H_{b}=-\left(\nabla+\frac{i B z}{2}\right)^{2}, \quad z \in R^{n}
$$

where $B$ is a real antisymmetric matrix. If $B$ is degenerated (this requires $n=2 d+l$ to be odd, and $2 d$ is the rank of the matrix $B$ ), then its eigenvalues have the form $\pm i b_{j}, j=1,2, \ldots, d$. In properly chosen coordinates $z=$ $\left(z_{1}, z_{2}, \ldots, z_{n}\right)=\left(x_{1}, y_{1}, x_{2}, y_{2}, \ldots, x_{d}, y_{d}, z_{2 d+1}, \ldots, z_{2 d+l}\right)$, the operator becomes

$$
H_{b}=-\sum_{j=1}^{d}\left(\left(\partial_{x_{j}}+i \frac{b_{j}}{2} y_{j}\right)^{2}+\left(\partial_{y_{j}}+i \frac{b_{j}}{2} x_{j}\right)\right)-\Delta_{l}
$$

where $\Delta_{l}=\sum_{j=1}^{l} \partial^{2} / \partial z_{2 d+j}^{2}$ is the Laplacian in $R^{l}$. The spectrum of $H_{b}$ is in the semiaxis starting from the point $c=\sum b_{j}$, and its spectral expansion is continuous (see [1]).

Let $b_{j}$ 's be positive. Denote by $E_{t}$ the spectral function of $H_{b}$. It is an integral operator with a kernel $e_{t}\left(z, z^{\prime}\right)$, which is skew-translation invariant; that is, $e_{t}\left(z+\omega, z^{\prime}+\omega\right)=$ $e_{t}\left(z, z^{\prime}\right) \exp i\left\langle B\left(z-z^{\prime}\right), \omega\right\rangle$.
For $\beta>0$, set the Riesz means of order $\beta$ as

$$
S_{\lambda}^{\beta} f(x)=\int_{0}^{\infty}\left(1-\frac{t}{\lambda}\right)_{+}^{\beta} d E_{t} f(x)=\int_{c}^{\infty}\left(1-\frac{t}{\lambda}\right)_{+}^{\beta} d E_{t} f(x),
$$

and the kernel of $S_{\lambda}^{\beta}$ as

$$
s_{\lambda}^{\beta}\left(z, z^{\prime}\right)=s^{\beta}\left(\lambda, z, z^{\prime}\right)=\int_{0}^{\infty}\left(1-\frac{t}{\lambda}\right)_{+}^{\beta} d e_{t}\left(z, z^{\prime}\right),
$$

with the same skew-translation invariance. We will denote by $S_{*}^{\beta}$ the corresponding maximal operator; that is,

$$
S_{*}^{\beta} f(x)=\sup _{0<\lambda<\infty}\left|S_{\lambda}^{\beta} f(x)\right| .
$$

As an indispensable part in harmonic analysis, many people investigate the convergence of Bochner-Riesz means for Fourier transform in norm and almost everywhere, which is defined as

$$
\left(T_{R}^{\beta} f\right)^{\wedge}(\xi)=\left(1-\frac{|\xi|^{2}}{R}\right)_{+}^{\beta} \hat{f}(\xi) .
$$

Since the convergence of $T_{R}^{\beta}$ in $L^{p}$-norm is equivalent to the boundedness of $T_{1}^{\beta}$ in $L^{P}\left(\mathbb{R}^{n}\right)$, persons look for the $L^{p}$ 
boundedness of it. For $2 n /(n+1+2 \beta)<p<2 n /(n-1-2 \beta)$ and $0 \leq \beta \leq(n-1 / 2)$, Carleson, Cordoba, and Fefferman turn out the boundedness in $\mathbb{R}^{2}$ (see [2-4]). When $n>2$, it is only proven for $\beta \geq(n-1) / 2(n+1)$ (see $[4,5])$. Returning the problem about almost everywhere convergence of $T_{R}^{\beta}$, Carbery has finished it for $\beta>0$ and $2 n /(n+1+2 \beta)<$ $p<2 n /(n-1-2 \beta)$ in two dimensions in 1983 (see [6]). For higher dimensions, it is completed by Christ only for $\beta \geq(n-1) / 2(n+1)$ (see [7]). In the special case, $\beta=0$, Fefferman studies the $L^{2}$-boundeness of $S_{\lambda}^{0}$ for $n \geq 2$ (see [8]). Not until 1988 was it solved by Carbery et al. for $\beta>0, n \geq 2$, and $2 \leq p<2 n /(n-1-2 \beta)$ (see [9]).

In [1], Rozenblum and Tashchiyan investigated the $L^{p_{-}}$ norm convergence for Riesz means for Schrödinger operator with constant magnetic fields. They showed that under the restriction theorem similar to one for Fourier transform in [5], it is of the same results as Bochner-Riesz means in $\mathbb{R}^{n}$. However, very few results are considered for almost everywhere convergence of Riesz means for Schrödinger operator with constant magnetic fields. In the paper, we are interested in it. Through researching the boundedness of the maximal operator in $L\left(\mathbb{R}^{n},|x|^{\alpha} d x\right)$, we get the desired result.

\section{Main Results}

Theorem 1. Set $\beta>0$ and $n \geq 2$. Write $p_{\beta}=2 n /(n-1-2 \beta)$. If $f \in L^{p}\left(\mathbb{R}^{n}\right)$ with $2 \leq p<p_{\beta}$, then $\lim _{\lambda \rightarrow \infty} S_{\lambda}^{\beta} f(x)=f(x)$ almost everywhere.

Usually, we replace the almost everywhere convergence of Riesz means with $L^{p}$ estimates for the maximal operator. However, we only need to think about weighted $L^{2}$ estimates for the maximal operator $S_{*}^{\beta}$, as follows. In fact, based on the idea in [9], for $2 \leq p<p_{\beta}$, there exists a number $\alpha$ with $0 \leq \alpha<1+2 \beta$ such that $L^{p} \subseteq L^{2}+L^{2}\left(|x|^{\alpha}\right)$. We have gotten $L^{2}$ boundedness of the maximal operator in another paper.

Theorem 2. Suppose that $\beta>0$ and $0 \leq \alpha<1+2 \beta \leq n$. Then,

$$
\int\left|S_{*}^{\beta} f(x)\right|^{2}|x|^{-\alpha} d x \leq C_{\alpha, \beta} \int|f(x)|^{2}|x|^{-\alpha} d x
$$

In order to prove the theorem, we introduce the following lemmas, which are the essential tools. In the following lemmas, we reduce the maximal operator to the one generated by a multiplier with compact support. Since it is easy to see that the boundedness of the maximal operator generated by the multipliers is independent of the index $\beta$, the dimension $n=2 d+l$ will not play an important role in the following estimates.

Lemma 3 (see [9]). If $0<\varepsilon<1 / 2$, then

$$
\int_{|| x|-1| \leq \varepsilon}|f(x)|^{2} d x \leq C_{\alpha} B_{\alpha}(\varepsilon) \int|f(x)|^{2}|x|^{\alpha} d x
$$

where

$$
B_{\alpha}(\varepsilon)= \begin{cases}\varepsilon^{\alpha}, \varepsilon|\log \varepsilon|, & 0 \leq \alpha<1, \\ \varepsilon|\log \varepsilon|, & \alpha=1, \\ \varepsilon, & 1<\alpha<n\end{cases}
$$

For a small $\delta>0$, let $m^{\delta}(t)$ be a $C^{\infty}$ function supported in $[1-\delta, 1]$ and satisfy

$$
0 \leq m^{\delta}(t) \leq 1, \quad\left|D^{k} m^{\delta}(t)\right| \leq C \delta^{-k} \quad \forall k \in \mathbb{N} .
$$

Define

$$
\begin{gathered}
K_{\lambda}^{\delta} f(x)=\int m^{\delta}(\lambda t) d E_{t} f(x), \\
K_{*}^{\delta} f(x)=\sup _{\lambda>0}\left|K_{\lambda}^{\delta} f(x)\right| .
\end{gathered}
$$

Let $\phi(t) \in C^{\infty}(\mathbb{R})$ supported in $[-1,2]$ and $\phi(t)=1$ for $0 \leq$ $t \leq 1$. Set

$$
h_{l}(t)= \begin{cases}\phi(t), & l=0 \\ \phi\left(2^{-l} t\right)-\phi\left(2^{1-l} t\right), & l \geq 1\end{cases}
$$

Denoting by $\chi_{E}$ the character function of the set $E$, write

$$
H_{j}(x, y)= \begin{cases}\chi_{[0,1)}(|x-y|), & j=0, \\ \chi_{\left[2^{j-1}, 2^{j}\right)}(|x-y|), & j \geq 1 .\end{cases}
$$

Let $k^{\delta}(t)$ be a function with Fourier transform as

$$
\widehat{k}_{\lambda}^{\delta}(t)=m^{\delta}(\lambda t) .
$$

Set

$$
\begin{gathered}
k_{\lambda}^{\delta, l, j}(t)=k_{\lambda}^{\delta}(t) h_{l}\left(\frac{\delta^{2} 2^{-j} t}{\lambda}\right), \\
E_{t}^{j} f(x)=\int_{\mathbb{R}^{n}} e_{t}(x, y) H_{j}(x, y) f(y) d y .
\end{gathered}
$$

Accordingly, define the operator $K_{\lambda}^{\delta, l, j}$ as

$$
K_{\lambda}^{\delta, l, j} f(x)=\int \widehat{k}_{\lambda}^{\delta, l, j}(t) d E_{t}^{j} f(x),
$$

where $\widehat{k}_{\lambda}^{\delta, l, j}$ is the Fourier transform of $k_{\lambda}^{\delta, l, j}$. Apparently, since

$$
K_{\lambda}^{\delta} f(x)=\int \hat{k}_{\lambda}^{\delta}(t) d E_{t} f(x),
$$

we decompose

$$
\begin{aligned}
K_{\lambda}^{\delta} f(x) & =\int \widehat{k}_{\lambda}^{\delta}(t) d E_{t} f(x) \\
& =\sum_{j=0}^{\infty} \sum_{l=0}^{\infty} K_{\lambda}^{\delta, l, j} f(x) \\
& =\sum_{j=0}^{\infty} \sum_{l=0}^{\infty} \int \hat{k}_{\lambda}^{\delta, l, j}(t) d E_{t}^{j} f(x) .
\end{aligned}
$$


Lemma 4. For $\lambda>0$, one has

$$
\left\|K_{\lambda}^{\delta, l, j} f(x)\right\|_{2}^{2} \leq C 2^{-2 M(j+l)} \delta^{2 M}\|f\|_{2}^{2},
$$

where the constant $C$ is independent of $\lambda$ and $\delta$.

Proof. With the method similar to the proof of Lemma 4 in [9], we write $h(t)=\phi(t)-\phi(2 t)$ and expand $m^{\delta}$ into a Taylor series around $\lambda t$. Then,

$$
\begin{aligned}
\widehat{k}_{\lambda}^{\delta, l, j}(t) & =\int m^{\delta}\left(\lambda\left(t-\frac{2^{-(j+l)} \delta^{2} r}{\lambda}\right)\right) \hat{h}(r) d r \\
& =\int m^{\delta}\left(\lambda t-2^{-(j+l)} \delta^{2} r\right) \hat{h}(r) d r \\
& =\int R_{M}(t, r) \hat{h}(r) d r,
\end{aligned}
$$

where the remainder $R_{M}$ satisfies

$$
\begin{aligned}
& \left|R_{M}(t, r)\right| \\
& \quad \leq\left|D^{M} m^{\delta}\right|\left|2^{-(j+l)} \delta^{2} r\right|^{M} \leq 2^{-M(l+j)} \delta^{M}|r|^{M} .
\end{aligned}
$$

But $\widehat{h}$ is a Schwartz function and can be integrated against $|r|^{M}$. Hence,

$$
\left|\widehat{k}_{\lambda}^{\delta, l, j}(t)\right| \leq C_{M^{2}} 2^{-M(j+l)} \delta^{M}
$$

Since $E_{\mathbb{R}}$ is a resolution of the identity, we see

$$
E_{\mathbb{R}} f(x)=\int_{0}^{\infty} d E_{t} f(x)=f(x) .
$$

Denote by $e_{\mathbb{R}}(x, y)$ the kernel of $E_{\mathbb{R}}$. For almost all $x_{0} \in$ $\mathbb{R}^{n}$, we let $S=\left\{y \in \mathbb{R}^{n}: e_{\mathbb{R}}\left(x_{0}, y\right) f(y)>0\right\}$. Decompose

$$
\begin{aligned}
f(x) & =f(x) \chi_{S}(x)+f(x) \chi_{\mathbb{R}^{n} / S}(x) \\
& =f_{1}(x)+f_{2}(x) .
\end{aligned}
$$

We have

$$
\begin{aligned}
\left|E_{\mathbb{R}}^{j} f\left(x_{0}\right)\right|= & \left|\int_{\mathbb{R}^{n}} e_{\mathbb{R}}\left(x_{0}, y\right) H_{j}\left(x_{0}, y\right) f(y) d y\right| \\
= & \mid \int_{\mathbb{R}^{n}} e_{\mathbb{R}}\left(x_{0}, y\right) H_{j}\left(x_{0}, y\right) f_{1}(y) d y \\
& +\int_{\mathbb{R}^{n}} e_{\mathbb{R}}\left(x_{0}, y\right) H_{j}\left(x_{0}, y\right) f_{2}(y) d y \mid \\
\leq & \left|\int_{\mathbb{R}^{n}} e_{\mathbb{R}}\left(x_{0}, y\right) H_{j}\left(x_{0}, y\right) f_{1}(y) d y\right| \\
& +\left|\int_{\mathbb{R}^{n}} e_{\mathbb{R}}\left(x_{0}, y\right) H_{j}\left(x_{0}, y\right) f_{2}(y) d y\right| \\
= & \int_{\mathbb{R}^{n}}\left|e_{\mathbb{R}}\left(x_{0}, y\right) H_{j}\left(x_{0}, y\right) f_{1}(y)\right| d y \\
& +\int_{\mathbb{R}^{n}}\left|e_{\mathbb{R}}\left(x_{0}, y\right) H_{j}\left(x_{0}, y\right) f_{2}(y)\right| d y \\
\leq & \int_{\mathbb{R}^{n}}\left|e_{\mathbb{R}}\left(x_{0}, y\right) f_{1}(y)\right| d y \\
& +\int_{\mathbb{R}^{n}}\left|e_{\mathbb{R}}\left(x_{0}, y\right) f_{2}(y)\right| d y \\
\leq & \left|\int_{\mathbb{R}^{n}} e_{\mathbb{R}}\left(x_{0}, y\right) f_{1}(y) d y\right| \\
& \left.+\left|f_{\mathbb{R}}\left(x_{0}\right)\right|+\mid x_{0}, y\right) f_{2}(y) d y \mid
\end{aligned}
$$

It is easy to show

$$
\begin{aligned}
& \left\|K_{\lambda}^{\delta, l, j} f(x)\right\|_{2}^{2} \\
& =\left\|\int \hat{k}_{\lambda}^{\delta, l, j}(t) d E_{t}^{j} f(x)\right\|_{2}^{2} \\
& =\left\|\int d E_{t}^{j}\left(\hat{k}_{\lambda}^{\delta, l, j}\left(H_{b}\right) f(x)\right)\right\|_{2}^{2} \\
& =\left\langle E_{\mathbb{R}}^{j}\left(\widehat{k}_{\lambda}^{\delta, l, j}\left(H_{b}\right) f\right), E_{\mathbb{R}}^{j}\left(\widehat{k}_{\lambda}^{\delta, l, j}\left(H_{b}\right) f\right)\right\rangle \\
& \leq\left\|\hat{k}_{\lambda}^{\delta, l, j}\left(H_{b}\right) f\right\|_{2}^{2} \\
& \leq \int_{0}^{\infty}\left|\widehat{k}_{\lambda}^{\delta, l, j}(t)\right|^{2} d\left(E_{t} f, f\right) \\
& \leq C_{M} 2^{-2 M(j+l)} \delta^{2 M} \int_{0}^{\infty} d\left(E_{t} f, f\right) \\
& \leq C_{M} 2^{-2 M(j+l)} \delta^{2 M}\|f\|_{2}^{2} .
\end{aligned}
$$


Lemma 5. If $0<\alpha<n$ and $\lambda>0$, then

$$
\int\left|\int \hat{k}_{\lambda}^{\delta}(t) d E_{t} f(x)\right|^{2} \frac{d x}{|x|^{\alpha}} \leq C_{\alpha} \delta \int|f(x)|^{2} \frac{d x}{|x|^{\alpha}},
$$

where $C_{\alpha}$ is independent of $\delta$ and $\lambda$.

Proof. Suppose that $f$ is supported in $\left\{|x| \leq C 2^{j}\right\}$. Write $f=$ $\chi_{\{0 \leq|x| \leq 1 / 4\}}(x) f(x)+\chi_{\left\{C<|x| \leq C 2^{j}\right\}}(x) f(x)+\chi_{\{1 / 4<|x| \leq C\}}(x) f(x)=$ $f_{1}+f_{2}+f_{3}$. If $C \leq 1 / 4$, then $f_{3}=0$. Since

$$
\begin{aligned}
& \left(\int\left|K_{\lambda}^{\delta} f(x)\right|^{2} \frac{d x}{|x|^{\alpha}}\right)^{1 / 2} \\
& =\left(\int\left|K_{\lambda}^{\delta}\left(f_{1}+f_{2}+f_{3}\right)(x)\right|^{2} \frac{d x}{|x|^{\alpha}}\right)^{1 / 2} \\
& \leq\left(\int\left|K_{\lambda}^{\delta} f_{1}\right|^{2} \frac{d x}{|x|^{\alpha}}\right)^{1 / 2}+\left(\int\left|K_{\lambda}^{\delta} f_{2}\right|^{2} \frac{d x}{|x|^{\alpha}}\right)^{1 / 2} \\
& \quad+\left(\int\left|K_{\lambda}^{\delta} f_{3}\right|^{2} \frac{d x}{|x|^{\alpha}}\right)^{1 / 2},
\end{aligned}
$$

we only need to prove that

$$
\begin{aligned}
& \int\left|\int \widehat{k}_{\lambda}^{\delta}(t) d E_{t} f_{i}(x)\right|^{2} \frac{d x}{|x|^{\alpha}} \\
& \quad \leq C_{\alpha} \delta \int|f(x)|^{2} \frac{d x}{|x|^{\alpha}}, \quad(i=1,2,3) .
\end{aligned}
$$

For the case of $i=2$, with Lemma 4, it follows that

$$
\begin{aligned}
& \int\left|K_{\lambda}^{\delta, j, l} f_{2}(x)\right|^{2} d x \\
& \leq C 2^{-l} 2^{-j M} \delta^{M}\left\|f_{2}\right\|_{2}^{2} \\
& \leq C 2^{-l} 2^{-j M} \delta^{M} \int_{C \leq|x| \leq C 2^{j}}|f(x)|^{2} d x \\
& \leq C 2^{-l} 2^{-j M} \delta^{M} \\
& \quad \times \sum_{k=0}^{j-1} \int_{C 2^{k} \leq|x| \leq C 2^{k+1}}|f(x)|^{2} d x .
\end{aligned}
$$

It is easy to see that

$$
\begin{aligned}
& \sum_{k=0}^{j-1} \int_{C 2^{k}<|x| \leq C 2^{k+1}}|f(x)|^{2} d x \\
& \quad \leq C \sum_{k=0}^{j-1} 2^{-k \alpha} \int_{C 2^{k}<|x| \leq C 2^{k+1}}|f(x)|^{2}|x|^{\alpha} d x \\
& \quad \leq C \int|f(x)|^{2}|x|^{\alpha} d x .
\end{aligned}
$$

Thus, we have

$$
\begin{aligned}
& \int\left|K_{\lambda}^{\delta, j, l} f_{2}(x)\right|^{2} d x \\
& \quad \leq C 2^{-l} 2^{-j M} \delta^{M} \int|f(x)|^{2}|x|^{\alpha} d x .
\end{aligned}
$$

Choosing $M>1$, we get

$$
\begin{aligned}
& \int\left|K_{\lambda}^{\delta, l, j} f_{2}(x)\right|^{2} d x \\
& \quad \leq C_{\alpha} 2^{-l} 2^{-j M} \delta \int|f(x)|^{2}|x|^{\alpha} d x .
\end{aligned}
$$

On the other hand, $E_{t}$ is self-adjoint. So,

$$
\begin{aligned}
& \left\langle E_{t} f, g\right\rangle \\
& \quad=\iint e_{t}(x, y) f(y) d y g(x) d x \\
& \quad=\int f(y) \int e_{t}(x, y) g(x) d x d y \\
& =\left\langle f, E_{t} g\right\rangle \\
& =\int f(y) \int e_{t}(y, x) g(x) d x d y .
\end{aligned}
$$

Hence,

$$
e_{t}(x, y)=e_{t}(y, x) \text {. }
$$

With

$$
H_{j}(x, y)=H_{j}(y, x)
$$

we get

$$
\begin{aligned}
e_{t}^{j}(x, y) & =e_{t}(x, y) H_{j}(x, y) \\
& =e_{t}(y, x) H_{j}(y, x)=e_{t}^{j}(y, x),
\end{aligned}
$$

and it implies that $K_{\lambda}^{\delta, l, j}$ is also self-adjoint; that is,

$$
\left\langle E_{t}^{j} f, g\right\rangle=\left\langle f, E_{t}^{j} g\right\rangle .
$$

Therefore, by duality,

$$
\begin{aligned}
& \int\left|K_{\lambda}^{\delta, l, j} f_{2}(x)\right|^{2} \frac{d x}{|x|^{\alpha}} \\
& \leq C_{\alpha} 2^{-l} 2^{-j M} \delta \int|f(x)|^{2} d x \\
& \leq C_{\alpha} 2^{-l} 2^{j(\alpha-M)} \delta \int|f(x)|^{2} \frac{d x}{|x|^{\alpha}} .
\end{aligned}
$$

Taking $M>\alpha+1$, we can establish the inequality

$$
\begin{aligned}
& \int\left|K_{\lambda}^{\delta, l, j} f_{2}(x)\right|^{2} \frac{d x}{|x|^{\alpha}} \\
& \leq C_{\alpha} 2^{-l} 2^{-j} \delta \int|f(x)|^{2} \frac{d x}{|x|^{\alpha}} .
\end{aligned}
$$

Nextly, we consider $i=1$. By the definition of $K_{\lambda}^{\delta, l, j}$ and $f_{1}$, we see that the kernel of $K_{\lambda}^{\delta, l, j}$ is supported in

$$
\left\{(x, y): 2^{j-1} \leq|x-y| \leq 2^{j}\right\}
$$


and $f(y)$ is supported in

$$
0 \leq|y| \leq \frac{1}{4}
$$

So, the support of $K_{\lambda}^{\delta, l, j} f(x)$ is contained in

$$
\left\{x \in \mathbb{R}^{n}: 2^{j}+\frac{1}{4} \geq|x| \geq 2^{j-1}-\frac{1}{4} \geq 2^{0-1}-\frac{1}{4}=\frac{1}{4}\right\} .
$$

With Lemma 4, we have

$$
\begin{aligned}
& \int\left|K_{\lambda}^{\delta, l, j} f_{1}(x)\right|^{2} \frac{d x}{|x|^{\alpha}} \\
& \leq \int\left|K_{\lambda}^{\delta, l, j} f_{1}(x)\right|^{2} 4^{\alpha} d x \\
& \leq C \int\left|K_{\lambda}^{\delta, l, j} f_{1}(x)\right|^{2} d x \\
& \leq C 2^{-l} 2^{-j M} \delta^{M}\left\|f_{1}\right\|_{2}^{2} \\
& \leq C 2^{-l} 2^{-j M} \delta^{M}\|f\|_{2}^{2} \\
& \leq C 2^{-l} 2^{-j M} \delta^{M}\left(2^{j}\right)^{\alpha} \int|f(x)|^{2} \frac{d x}{|x|^{\alpha}} \\
& \leq C 2^{-l} 2^{-j} \delta \int|f(x)|^{2} \frac{d x}{|x|^{\alpha}},
\end{aligned}
$$

where $M>\alpha+1$.

At last, we turn to the case of $i=3$. Similar to the aforementioned, we have

$$
\begin{aligned}
& \int\left|K_{\lambda}^{\delta, l, j} f_{3}(x)\right|^{2} d x \\
& \leq C 2^{-l} 2^{-j M} \delta^{M}\left\|f_{3}\right\|_{2}^{2} \\
& \leq C 2^{-l} 2^{-j M} \delta^{M} \int_{1 / 4 \leq|x| \leq C}|f(x)|^{2} d x \\
& \leq C 2^{-l} 2^{-j M} \delta^{M}\left(\frac{1}{4}\right)^{-\alpha} \int_{1 / 4 \leq|x| \leq C}|f(x)|^{2}\left(\frac{1}{4}\right)^{\alpha} d x \\
& \leq C 2^{-l} 2^{-j M} \delta^{M} 4^{\alpha} \int_{1 / 4 \leq|x| \leq C}|f(x)|^{2}|x|^{\alpha} d x \\
& \leq C 2^{-l} 2^{-j M} \delta^{M} \int|f(x)|^{2}|x|^{\alpha} d x .
\end{aligned}
$$

By duality again, we see

$$
\begin{aligned}
& \int\left|K_{\lambda}^{\delta, l, j} f_{3}(x)\right|^{2} \frac{d x}{|x|^{\alpha}} \\
& \leq C 2^{-l} 2^{-j M} \delta^{M} \int|f(x)|^{2} d x \\
& \leq C 2^{-l} 2^{-j M} \delta^{M}\left(2^{j}\right)^{\alpha} \int|f(x)|^{2} \frac{d x}{|x|^{\alpha}} \\
& \leq C 2^{-l} 2^{-j(M-\alpha)} \delta^{M} \int|f(x)|^{2} \frac{d x}{|x|^{\alpha}}
\end{aligned}
$$

Through we choose $M>\alpha+1$, it is not difficult to get

$$
\begin{aligned}
& \int\left|K_{\lambda}^{\delta, l, j} f_{3}(x)\right|^{2} \frac{d x}{|x|^{\alpha}} \\
& \leq C 2^{-l} 2^{-j} \delta \int|f(x)|^{2} \frac{d x}{|x|^{\alpha}} .
\end{aligned}
$$

Combining (28), (40), (44) with (47), when $f$ is supported in $\left\{|x| \leq C 2^{j}\right\}$, we come to the conclusion

$$
\begin{aligned}
& \int\left|K_{\lambda}^{\delta, l, j} f(x)\right|^{2} \frac{d x}{|x|^{\alpha}} \\
& \leq C 2^{-l} 2^{-j} \delta \int|f(x)|^{2} \frac{d x}{|x|^{\alpha}}
\end{aligned}
$$

Now, we hope to establish (48) for all $f \in L^{2}\left(\mathbb{R}^{n}\right)$. Decompose $f=\sum_{i \in \mathbb{Z}^{n}} \chi_{Q_{i}} f=\sum_{i \in \mathbb{Z}^{n}} f_{i}$, where $\left\{Q_{i}\right\}$ are disjoint cubes of common side $10 \cdot 2^{j}$ with $Q_{0}$ centered at 0 . Since $\left\{K_{\lambda}^{\delta, l, j} f_{i}(x)\right\}$ have essentially disjoint supports, it suffices to prove (48) for every $f_{i}$. When $i=0$, we have proved it. If $i>1$, then

$$
\begin{aligned}
0 & <\left(\frac{1}{2}+l\right) 10 \cdot 2^{j} \\
& <|x|^{-\alpha}<\left(\frac{1}{2}+l+1\right) 10 \cdot 2^{j} \quad(l \geq 0) .
\end{aligned}
$$

Therefore, we only need to confirm

$$
\int\left|K_{\lambda}^{\delta, l, j} f(x)\right|^{2} d x \leq C_{\alpha} 2^{-l} 2^{-j} \delta \int|f(x)|^{2} d x .
$$

In fact, it follows from Lemma 4 that

$$
\begin{aligned}
& \int\left|K_{\lambda}^{\delta, l, j} f(x)\right|^{2} d x \\
& \leq C 2^{-2 M(j+l)} \delta^{2 M} \int|f(x)|^{2} d x \\
& \leq C 2^{-l} 2^{-j} \delta \int|f(x)|^{2} d x .
\end{aligned}
$$

At present, we complete the proof of Lemma 5.

Lemma 6. For $\delta>0, k \in \mathbb{Z}$, and $0 \leq \alpha<n$, one gets

$$
\int_{\mathbb{R}^{n}} \int_{2^{k-1}}^{2^{k}}\left|K_{\lambda}^{\delta} f(x)\right|^{2} \frac{d \lambda}{\lambda} \frac{d x}{|x|^{\alpha}} \leq C_{\alpha} \delta \int|f(x)|^{2} \frac{d x}{|x|^{\alpha}}
$$


Proof. Applying Minkowski and Cauchy-Schwartz's inequalities into the left hand side of (52), we get

$$
\begin{aligned}
& \int_{\mathbb{R}^{n}}\left|\int_{2^{k-1}}^{2^{k}} K_{\lambda}^{\delta} f(x) \frac{d \lambda}{\lambda}\right|^{2}|x|^{-\alpha} d x \\
& \leq C\left(\int_{2^{k-1}}^{2^{k}}\left(\int_{\mathbb{R}^{n}}\left|K_{\lambda}^{\delta} f(x)\right|^{2}|x|^{-\alpha} d x\right)^{1 / 2} \frac{d \lambda}{\lambda}\right)^{2} \\
& \leq C\left(\left(\int_{2^{k-1}}^{2^{k}} \int_{\mathbb{R}^{n}}\left|K_{\lambda}^{\delta} f(x)\right|^{2}|x|^{-\alpha} d x \frac{d \lambda}{\lambda}\right)^{1 / 2}\right. \\
& \left.\quad \leq C \int_{2^{k-1}}^{2^{k}} \int_{\mathbb{R}^{n}}\left|\int_{\lambda}^{2^{k}} f(x)\right|^{2}|x|^{-\alpha} d x \frac{d \lambda}{\lambda}\right)^{2}
\end{aligned}
$$

Now, it suffices to prove that

$$
\begin{aligned}
& \int_{\mathbb{R}^{n}}\left|K_{\lambda}^{\delta} f(x)\right|^{2}|x|^{-\alpha} d x \\
& \quad \leq C_{\alpha} \delta \int_{\mathbb{R}^{n}}|f(x)|^{2}|x|^{-\alpha} d x,
\end{aligned}
$$

where $C_{\alpha} \delta$ is uniform in $2^{k-1} \leq \lambda \leq 2^{k}$. For $0 \leq \alpha<n$, it is equivalent to

$$
\int_{\mathbb{R}^{n}}\left|K_{\lambda}^{\delta} g(x)\right|^{2} \frac{d x}{|x|^{\alpha}} \leq C_{\alpha} \delta \int_{\mathbb{R}^{n}}|g(x)|^{2} \frac{d x}{|x|^{\alpha}} .
$$

It is just as the result of Lemma 5.

Now, we come back to the proof of Theorem 2.

Proof. As in [3], we can decompose

$$
\left(1-\frac{t}{\lambda}\right)_{+}^{\beta}=\sum_{k=0}^{\infty} 2^{-k \beta} m^{2^{-k}}\left(\frac{t}{\lambda}\right) .
$$

Thus,

$$
S_{*}^{\beta} f(x) \leq \sum_{k=0}^{\infty} 2^{-k \beta} K_{*}^{2^{-k}} f(x) .
$$

Consequently, we consider

$$
\int_{\mathbb{R}^{n}}\left|K_{*}^{\delta} f(x)\right|^{2} \frac{d x}{|x|^{\alpha}} \leq C_{\alpha} \delta \int_{\mathbb{R}^{n}}|f(x)|^{2} \frac{d x}{|x|^{\alpha}} .
$$

Let

$$
G^{\delta} f(x)=\left(\int_{0}^{\infty}\left|K_{\lambda}^{\delta} f(x)\right|^{2} \frac{d \lambda}{\lambda}\right)^{1 / 2}
$$

and $\bar{G}^{\delta}$ be defined in the same way but using instead of $m^{\delta}$ the function

$$
\bar{m}^{\delta}(\lambda)=\delta \lambda \frac{d m^{\delta}(\lambda)}{\lambda}
$$

which satisfies the same estimates as $m^{\delta}$. Then, by the fundamental theorem in calculus and Hölder's inequality, we have

$$
\begin{aligned}
\left|K_{*}^{\delta} f(x)\right|^{2} \leq & \int_{0}^{\infty} 2\left|K_{\lambda}^{\delta} f(x) \frac{d K_{\lambda}^{\delta} f(x)}{d \lambda}\right| d \lambda \\
\leq & 2 \delta^{-1} \int_{0}^{\infty} \frac{\left|K_{\lambda}^{\delta} f(x)\right|}{\lambda^{1 / 2}} \lambda \delta \frac{\left|d K_{\lambda}^{\delta} f(x) / d \lambda\right|}{\lambda^{1 / 2}} d \lambda \\
\leq & 2 \delta^{-1}\left(\int_{0}^{\infty}\left|K_{\lambda}^{\delta} f(x)\right|^{2} \frac{d \lambda}{\lambda}\right)^{1 / 2} \\
& \times\left(\int_{0}^{\infty}\left|\lambda \delta \frac{d K_{\lambda}^{\delta} f(x)}{d \lambda}\right|^{2} \frac{d \lambda}{\lambda}\right)^{1 / 2} \\
= & 2 \delta^{-1} G^{\delta} f(x) \bar{G} f(x) .
\end{aligned}
$$

Take a Schwartz function $\psi$ such that $\psi(0)=0$ and $\psi(t)=$ 1 if $1 / 2 \leq t \leq 2$ and $\psi_{k}(t)=\psi\left(2^{k} t\right)$. Then, when $2^{k-1} \leq \lambda \leq$ $2^{k}$, we have

$$
K_{\lambda}^{\delta} f(x)=\int m^{\delta}(\lambda t) \psi_{k}(t) d E_{t} f(x)
$$

Using Lemma 6, we get

$$
\begin{aligned}
& \int_{\mathbb{R}^{n}} \int_{2^{k-1}}^{2^{k}}\left|K_{\lambda}^{\delta} f(x)\right|^{2} \frac{d \lambda}{\lambda} \frac{d x}{|x|^{\alpha}} \\
& \quad=\int_{\mathbb{R}^{n}} \int_{2^{k-1}}^{2^{k}}\left|\int m^{\delta}(\lambda t) \psi_{k}(t) d E_{t} f(x)\right|^{2} \frac{d \lambda}{\lambda} \frac{d x}{|x|^{\alpha}} \\
& \quad=\int_{\mathbb{R}^{n}} \int_{2^{k-1}}^{2^{k}}\left|\int m^{\delta}(\lambda t) d E_{t}\left(\psi_{k}\left(H_{b}\right) f\right)(x)\right|^{2} \frac{d \lambda}{\lambda} \frac{d x}{|x|^{\alpha}} \\
& \leq C_{\alpha} \delta \int\left|\psi_{k}\left(H_{b}\right) f(x)\right|^{2} \frac{d x}{|x|^{\alpha}} \\
& \quad=C_{\alpha} \delta \int\left|\int \psi_{k}(t) d E_{t} f(x)\right|^{2} \frac{d x}{|x|^{\alpha}} .
\end{aligned}
$$

From Theorem 3.1 in page 411 of [10] and the density of $L_{c}^{\infty}$ in $L^{p}(w)$, we induce that

$$
\left(\sum_{k=-\infty}^{\infty}\left|\int \psi_{k}(t) d E_{t} f(x)\right|^{2}\right)^{1 / 2}
$$


is bounded in $L^{2}\left(d x /|x|^{\alpha}\right)$. As a result,

$$
\begin{aligned}
\int\left(G^{\delta} f(x)\right)^{2} \frac{d x}{|x|^{\alpha}} & =\iint_{0}^{\infty}\left|K_{\lambda}^{\delta} f(x)\right|^{2} \frac{d \lambda}{\lambda} \frac{d x}{|x|^{\alpha}} \\
& =\int \sum_{k=-\infty}^{\infty} \int_{2^{k-1}}^{2^{k}}\left|K_{\lambda}^{\delta} f(x)\right|^{2} \frac{d \lambda}{\lambda} \frac{d x}{|x|^{\alpha}} \\
& =\sum_{k=-\infty}^{\infty} \iint_{2^{k-1}}^{2^{k}}\left|K_{\lambda}^{\delta} f(x)\right|^{2} \frac{d \lambda}{\lambda} \frac{d x}{|x|^{\alpha}} \\
& \leq \sum_{k=-\infty}^{\infty} C_{\alpha} \delta \int\left|\int \psi_{k}(t) d E_{t} f(x)\right|^{2} \frac{d x}{|x|^{\alpha}} \\
& \leq C_{\alpha} \delta \int \sum_{k=-\infty}^{\infty}\left|\int \psi_{k}(t) d E_{t} f(x)\right|^{2} \frac{d x}{|x|^{\alpha}} \\
& \leq C_{\alpha} \delta \int|f(x)|^{2} \frac{d x}{|x|^{\alpha}} .
\end{aligned}
$$

At last, with (61) and Hölder's inequality, we come to the result that

$$
\int\left(K_{*}^{\delta} f(x)\right)^{2} \frac{d x}{|x|^{\alpha}} \leq C_{\alpha} \int|f(x)|^{2} \frac{d x}{|x|^{\alpha}} .
$$

\section{Acknowledgments}

The authors deeply thank the referee for reviewing their paper. The first author was supported by the NSF of China (71201051, 71031004, and 71073047), NSF of Hunan Province (2012RS4028) and Postdoctoral Science Foundation of China (2012M521513). The second author was supported by the NSF of China (10771054) and NSF of Hunan Province (09JJ5002).

\section{References}

[1] G. Rozenblum and G. Tashchiyan, "Riesz $L^{p}$ summability of spectral expansions related to the Schrödinger operator with constant magnetic field," Journal of Mathematical Analysis and Applications, vol. 284, no. 1, pp. 315-331, 2003.

[2] L. Carleson and P. Sjölin, "Oscillatory integrals and a multiplier problem for the disc," Studia Mathematica, vol. 44, pp. 287-299, 1972.

[3] A. Cordoba, "The Kakeya maximal function and the spherical summation multipliers," American Journal of Mathematics, vol. 99, no. 1, pp. 1-22, 1977.

[4] C. Fefferman, "A note on spherical summation multipliers," Israel Journal of Mathematics, vol. 15, pp. 44-52, 1973.

[5] P. A. Tomas, "A restriction theorem for the Fourier transform," Bulletin of the American Mathematical Society, vol. 81, pp. 477478, 1975.

[6] A. Carbery, "The boundedness of the maximal Bochner-Riesz operator on $L^{4}\left(\mathbb{R}^{2}\right)$," Duke Mathematical Journal, vol. 50, no. 2 , pp. 409-416, 1983.

[7] M. Christ, "On almost everywhere convergence of BochnerRiesz means in higher dimensions," Proceedings of the American Mathematical Society, vol. 95, no. 1, pp. 16-20, 1985.
[8] C. Fefferman, “The multiplier problem for the ball," Annals of Mathematics, vol. 94, pp. 330-336, 1971.

[9] A. Carbery, J. L. Rubio de Francia, and L. Vega, "Almost everywhere summability of Fourier integrals," Journal of the London Mathematical Society, vol. 38, no. 3, pp. 513-524, 1988.

[10] J. Garcia-Cuerva and J. L. Rubio De Francia, Weighted Norm Inequalities and Related Topics, North-Holland, Amsterdam, The Netherlands, 1985. 


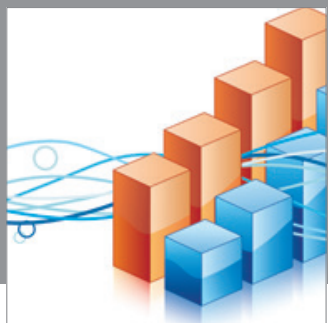

Advances in

Operations Research

mansans

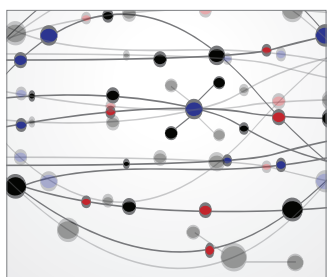

The Scientific World Journal
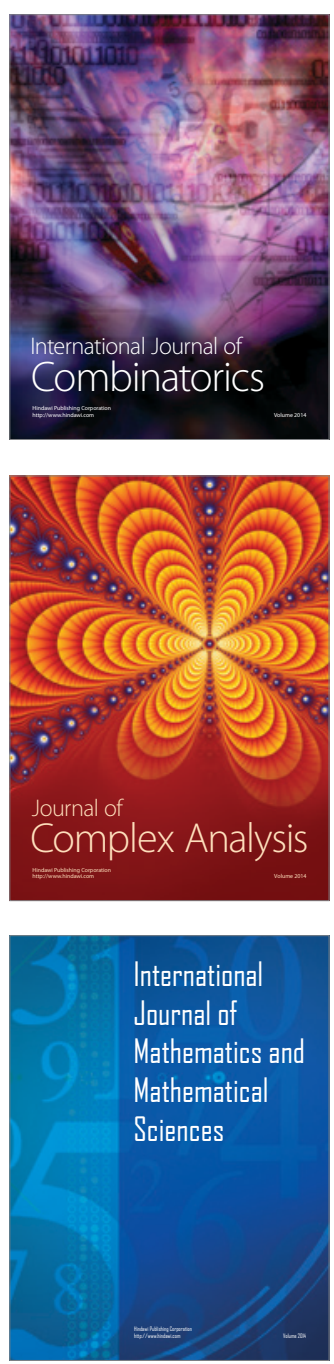
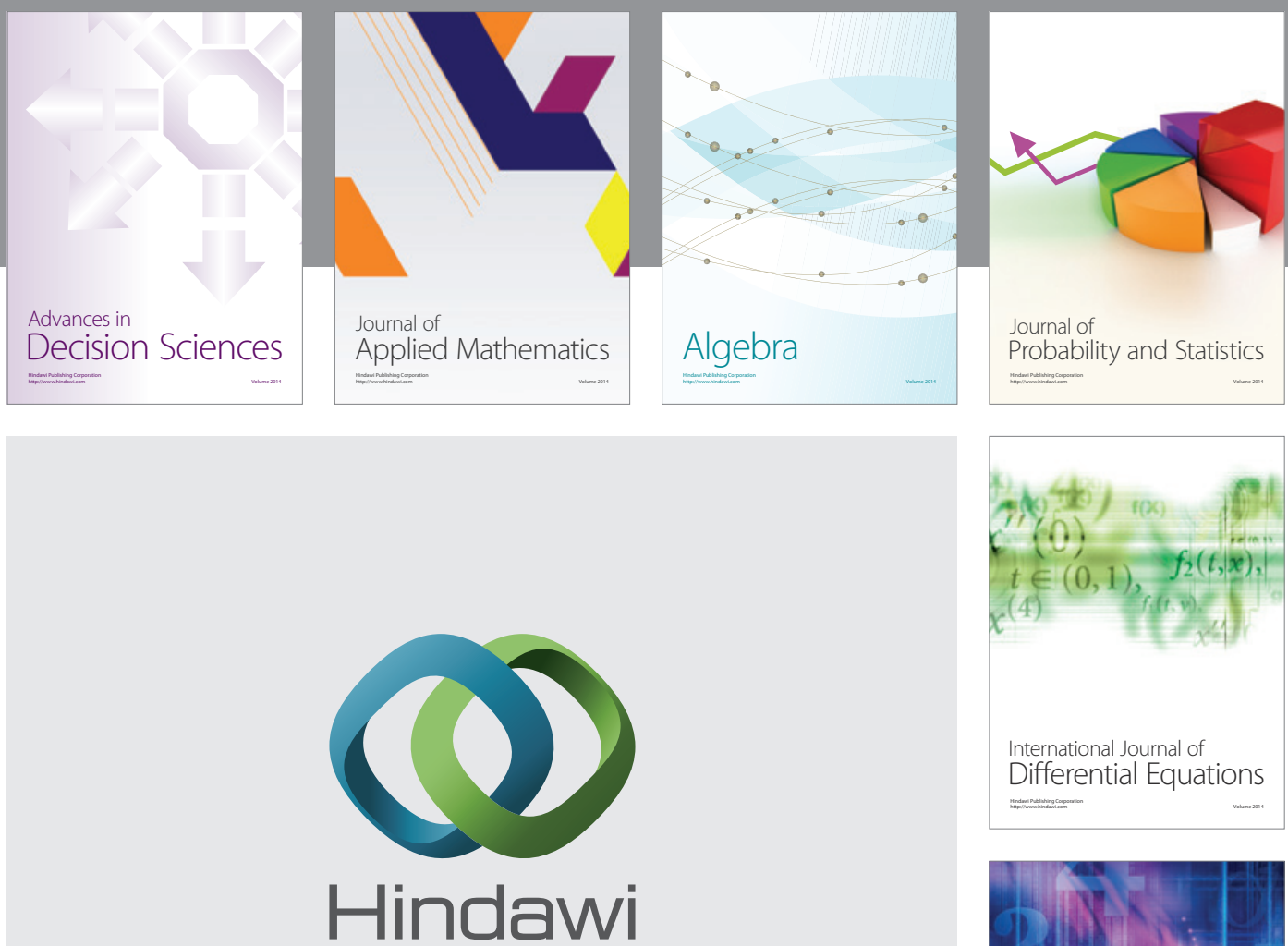

Submit your manuscripts at http://www.hindawi.com
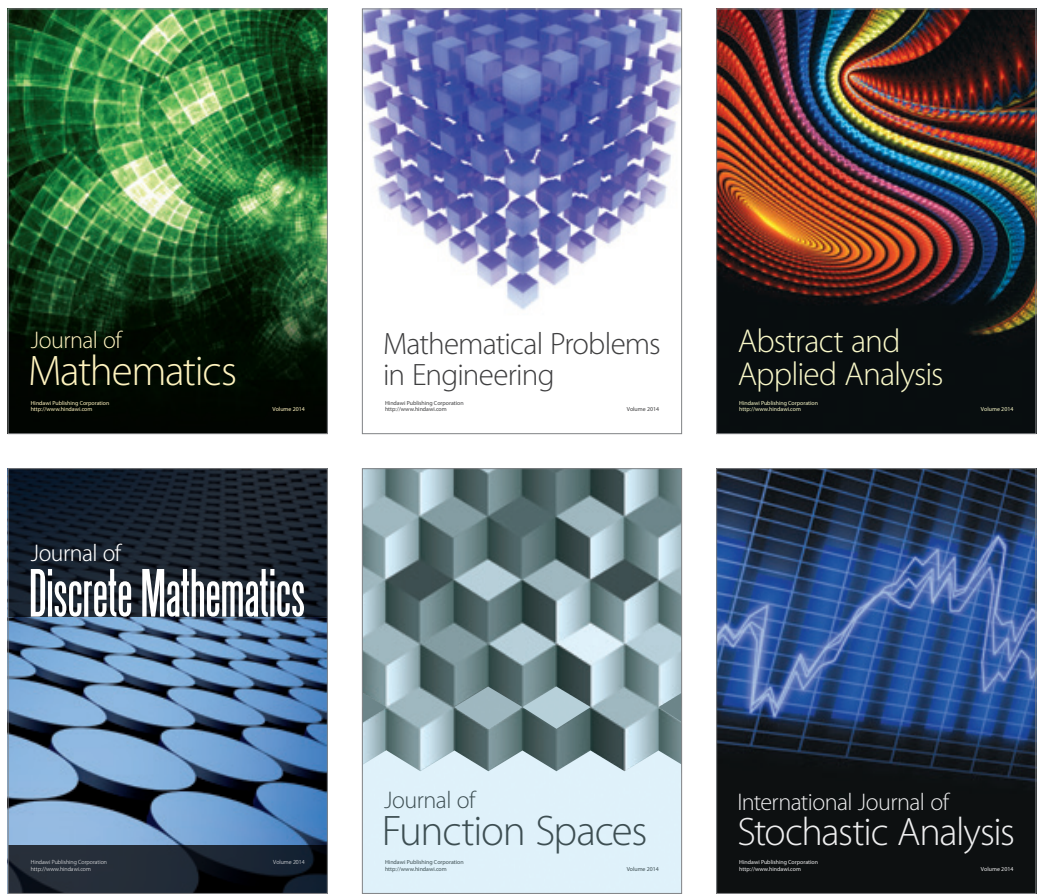

Journal of

Function Spaces

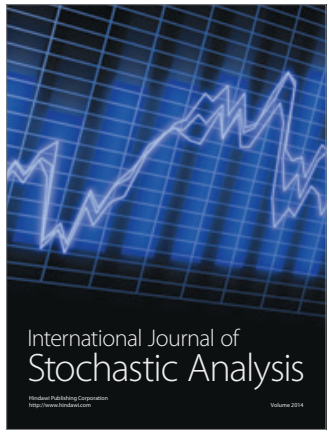

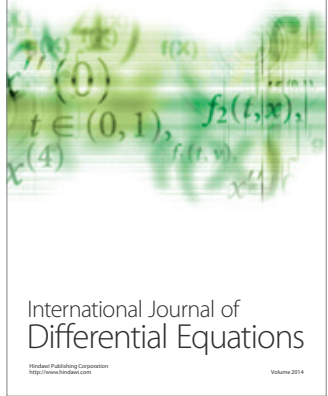
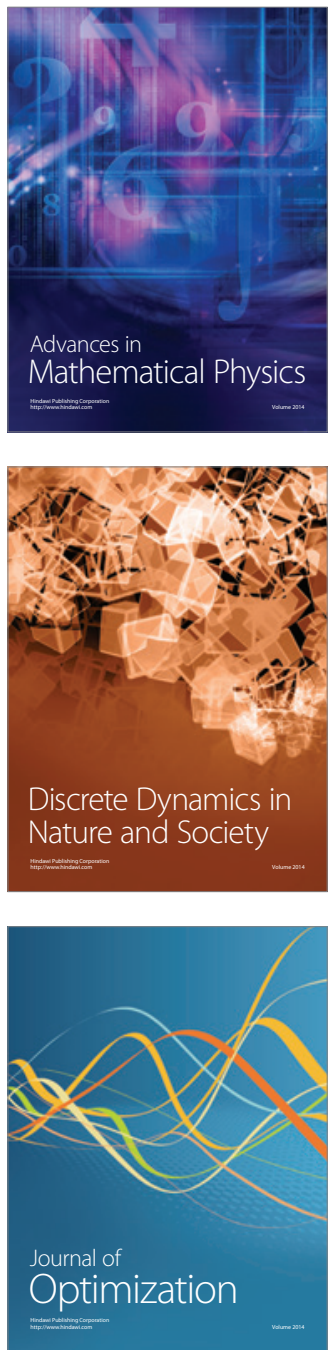